\title{
Constructing a sequence of random walks strongly converging to Brownian motion
}

\author{
Philippe Marchal \\ CNRS and École normale supérieure, 45 rue d'Ulm, 75005 Paris, France \\ Philippe. Marchaleens. fr
}

We give an algorithm which constructs recursively a sequence of simple random walks on $\mathbb{Z}$ converging almost surely to a Brownian motion. One obtains by the same method conditional versions of the simple random walk converging to the excursion, the bridge, the meander or the normalized pseudobridge.

Keywords: strong convergence, simple random walk, Brownian motion

\section{Introduction}

It is one of the most basic facts in probability theory that random walks, after proper rescaling, converge to Brownian motion. However, Donsker's classical theorem [Don51] only states a convergence in law. Various results of almost sure convergence exist (see e.g. [KMT75, KMT76] and the references therein) but involves rather intricate relations between the converging sequence of random walks and the limiting Brownian motion.

The aim of this paper is to give an explicit algorithm constructing a sequence of simple random walks on $\mathbb{Z}$ converging to a Brownian motion. This algorithm is described in the next section and leads to the following

Theorem 1 There exist on a common probability space a family $\left(S^{n}, n \geq 1\right)$ of random walks on $\mathbb{Z}$ and a linear Brownian motion $\left(B_{t}, 0 \leq t \leq 1\right)$ such that:

(i) for every $n, S^{n}$ has the law of a simple random walk with $n$ steps starting at 0 ,

(ii) almost surely, for every $t \in[0,1]$, as $n \rightarrow \infty$,

$$
\frac{S_{[n t]}^{n}}{\sqrt{n}} \rightarrow B_{t} .
$$

An important feature of our construction is that it can be adapted to conditional versions of the random walk, yielding the following generalization:

Theorem 2 There exists a family $\left(S^{n}, n \geq 1\right)$ of random walks on $\mathbb{Z}$ starting at 0 where for every $n, S^{n}$ respectively:

(1) has length $2 n$ and is conditioned to return to 0 at time $2 n$,

1365-8050 @ 2003 Discrete Mathematics and Theoretical Computer Science (DMTCS), Nancy, France 
(2) has length $2 n$, is conditioned to return to 0 at time $2 n$ and to stay positive from time 1 to $2 n-1$,

(3) has length $n$ and is conditioned to stay positive from time 1 to $n-1$,

and such that almost surely, for every $t \in[0,1], S_{[2 n t]}^{n} / \sqrt{n}$ (or $S_{[n t]}^{n} / \sqrt{n}$ in the third case) converges to $\widetilde{B}_{t}$ where $\left(\widetilde{B}_{t} 0 \leq t \leq 1\right)$ is respectively:

(1) a Brownian bridge,

(2) a Brownian excursion,

(3) a Brownian meander.

A similar result holds for random walks biased by their local time at 0 and converging almost surely to the Brownian pseudobridge.

Theorem 3 There exists a family $\left(S^{n}, n \geq 1\right)$ of random walks on $\mathbb{Z}$ of length $2 n$ with $S_{0}^{n}=S_{2 n}^{n}=0$ such that:

(a) for every path $\mathcal{P}$ of length $2 n$ satisfying $\mathcal{P}_{0}=\mathcal{P}_{2 n}=0, \mathbb{P}\left(S^{n}=\mathscr{P}\right)$ is proportional to $1 / L^{0}(\mathcal{P})$ where $L^{0}(\mathcal{P})$ is the number of visits of 0 of $\mathcal{P}$,

(b) almost surely, for every $t \in[0,1], S_{[2 n t]}^{n} / \sqrt{n}$ converges to some real $\widehat{B}_{t}$ where $\left(\widehat{B}_{t}, 0 \leq t \leq 1\right)$ is a normalized Brownian pseudobridge.

Our algorithms construct $S^{n}$ recursively and are therefore interruptible. Notice that even if one does not want strong convergence, the natural method to construct a discrete version of the Brownian pseudobridge consists in running a random walk up to $T_{N}$, the $N$-th return time to 0 , for large $N$, and then rescaling. But this method has the major drawback that $E\left(T_{N}\right)=\infty$.

It is well-known in combinatorics that there exist natural bijections between binary trees and excursions of the simple random walk. In this context, the construction we shall describe in case (2) of Theorem 2 is a pathwise counterpart of the tree-generating algorithm introduced in [Rém85] and which can be described simply as follows. Start with a tree with just a root and two children of the root. Then recursively: choose a random edge $e$, split it into two edges $e_{1}$ and $e_{2}$ with a vertex $v$ between $e_{1}$ and $e_{2}$, and add new edge $e_{3}$ connected only to $v$, either to the right or to the left of $e_{2}$. It is obvious that one generates this way random binary trees with the uniform distribution.

Finally, let us mention that the convergence rate for our construction can be bounded by

$$
\mathbb{E}\left[\int_{0}^{1}\left|\frac{S_{[t n]}^{n}}{\sqrt{n}}-B_{t}\right| d t\right] \leq \frac{c}{n^{1 / 4}}
$$

for some constant $c>0$. This indicates a slower convergence rate than the optimal one, which is $O(\log n / \sqrt{n})$ and is achieved in [KMT75].

The algorithms generating the converging sequences of random walks $\left(S^{n}\right)$ are described in the next section. The proofs of Theorems 1 and 2 are sketched in Section 3. Further results and the proof of Theorem 3 are given in Section 4.

\section{Construction of the random walks}

\subsection{Some terminology}

Recall that an excursion is a part of a path between two consecutive zeros and that the meander is the part of the path after the last zero. If the meander of a path $\mathcal{P}$ is positive, a point $t$ in the meander is visible 

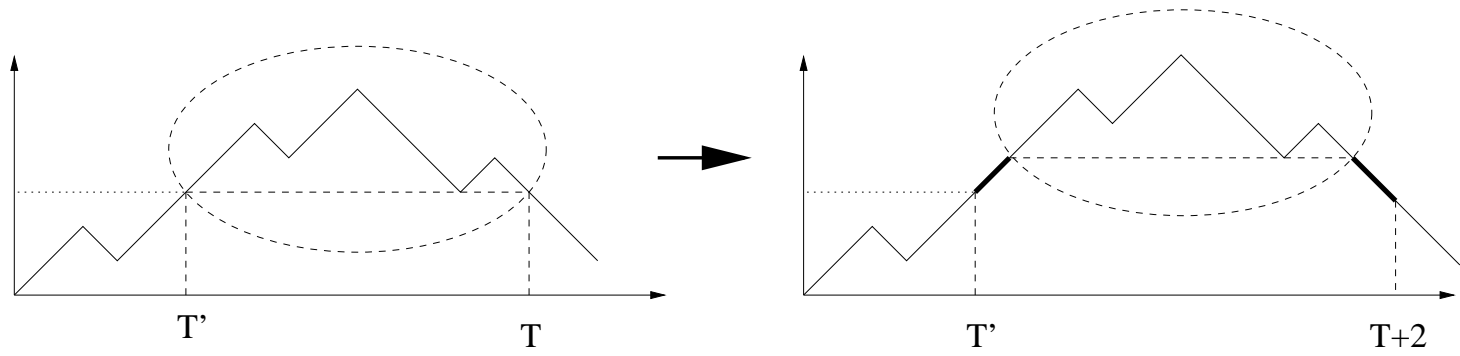

Fig. 1: Lifting the Dyck path before time T

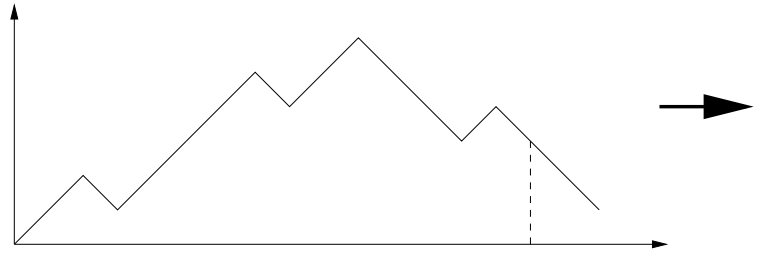

$\mathrm{T}$

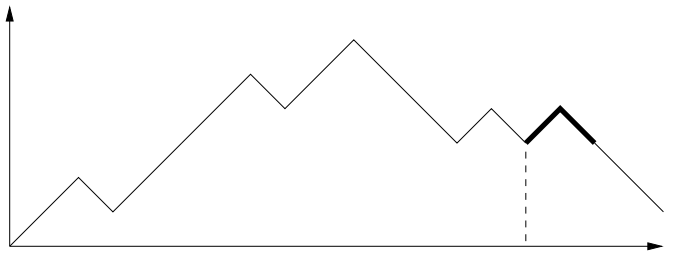

$\mathrm{T}$

Fig. 2: Inserting a hat at time $\mathrm{T}$

from the right if

$$
\mathcal{P}(t)=\min \{\mathcal{P}(n), n \geq t\} .
$$

Finally, we call a positive hat a sequence of a positive step followed by a negative step. A negative hat is defined likewise.

Let us describe a procedure to extend a path $\mathcal{P}$. Suppose that $\mathcal{P}(T)>0$. Then lifting the Dyck path before time $T$ means the following. Let

$$
T^{\prime}=1+\sup \{n \leq T, \mathcal{P}(n)<\mathcal{P}(T)\}
$$

Then form the new path $\mathcal{P}^{\prime}$ by inserting a positive step at time $T^{\prime}$ and then a negative step at time $T+1$. Remark that if $\mathcal{P}(T-1)=\mathcal{P}(T)-1$, then $T^{\prime}=T$ and lifting the Dyck path before time $T$ amounts to inserting a hat at time $T$.

\subsection{The algorithm for Theorem 1}

We shall in fact describe an algorithm generating $S^{n}:=S^{2 n-1}$. One constructs $S^{2 n}$ by adding a last random step to $\mathcal{S}^{n}$. First choose $\mathcal{S}^{1}$ at random. Then to generate $\mathcal{S}^{n+1}$ from $\mathcal{S}^{n}$ :

I. Choose a random time $t$ uniformly on $\{0, \ldots 2 n-1\}$.

II. If $S^{n}(t)=0$, insert a positive or negative hat at time $t$ with respective probabilities $1 / 2-1 / 2$. 
III. If $t$ is in a positive excursion,

1. with probability $1 / 2$ insert a positive hat at time $t$.

2. with probability $1 / 2$ lift the Dyck path before time $t$.

IV. If $t$ is in the meander of $S^{n}$ and if this meander is positive,

1. If $t$ is invisible from the right, or if $t$ is visible from the right and $\mathcal{S}^{n}(t)$ is even, proceed as in III.

2. If $t$ is visible from the right and $\mathcal{S}^{n}(t)$ is odd,

a. with probability $1 / 2$ insert at time $t$ a positive hat,

b. with probability $1 / 2$ insert at time $t$ two positive steps.

If $t$ is in a negative excursion or in a negative meander, the procedure is the exact analogue of III or IV. Of course, all the choices are assumed independent.

\subsection{The algorithms for Theorems 2 and 3}

In case (1) of Theorem 2, begin with $S^{0}$ the empty path. To obtain $S^{n+1}$ from $S^{n}$, choose a random time $t$ uniformly in $\{0, \ldots 2 n\}$ and apply procedure II or III.

In case (2) of Theorem 2, begin with $S^{1}$ a positive hat. To obtain $S^{n+1}$ from $S^{n}$, choose a random time $t$ uniformly in $\{1, \ldots 2 n-1\}$ and apply procedure III.

In case (3) of Theorem 2 begin with $S^{1}$ a positive step. Then to obtain $S^{2 n+1}$ from $S^{2 n-1}$, choose a random time $t$ uniformly in $\{1, \ldots 2 n-1\}$ and apply procedure $\mathbf{I V}$. To obtain $S^{2 n}$ from $S^{2 n-1}$, just add a last random step. Remark that in each case, the algorithm of Theorem 2 is just the restriction of the algorithm of Theorem 1 to the subpart of the path we are considering.

Finally for Theorem 3 , begin with $S^{1}$ a positive hat. To obtain $S^{n+1}$ from $S^{n}$, choose a random time $t \in\{0,1, \ldots 2 n\}$ with probability proportional to

- 1 if $S^{n}(t)>0$

- $1-1 / k$ if $S^{n}(t)=0$, where $k$ denotes the number of zeros of $S^{n}$. This includes the first and the last zero, so for instance $k=2$ in the beginning.

Then apply procedure II or III.

Remarks. In Case (2) of Theorem 2, it should be clear that the algorithm is just a translation of the treegenerating algorithm described in the introduction, for a suitable choice of the bijection between Dyck paths and binary trees. In particular, in Rémy's algorithm, one has to choose between putting the new edge $e_{3}$ to the right or to the left of $e_{2}$. In the algorithm of Theorem 2, this corresponds to choosing between lifting a Dyck path and inserting a hat. In some cases, both choices are equivalent, as noticed in Section 2.1. This corresponds to the fact that in Rémy's algorithm, if the edge $e_{2}$ is connected to a leaf, putting $e_{3}$ to the right or to the left of $e_{2}$ yields the same binary tree. 


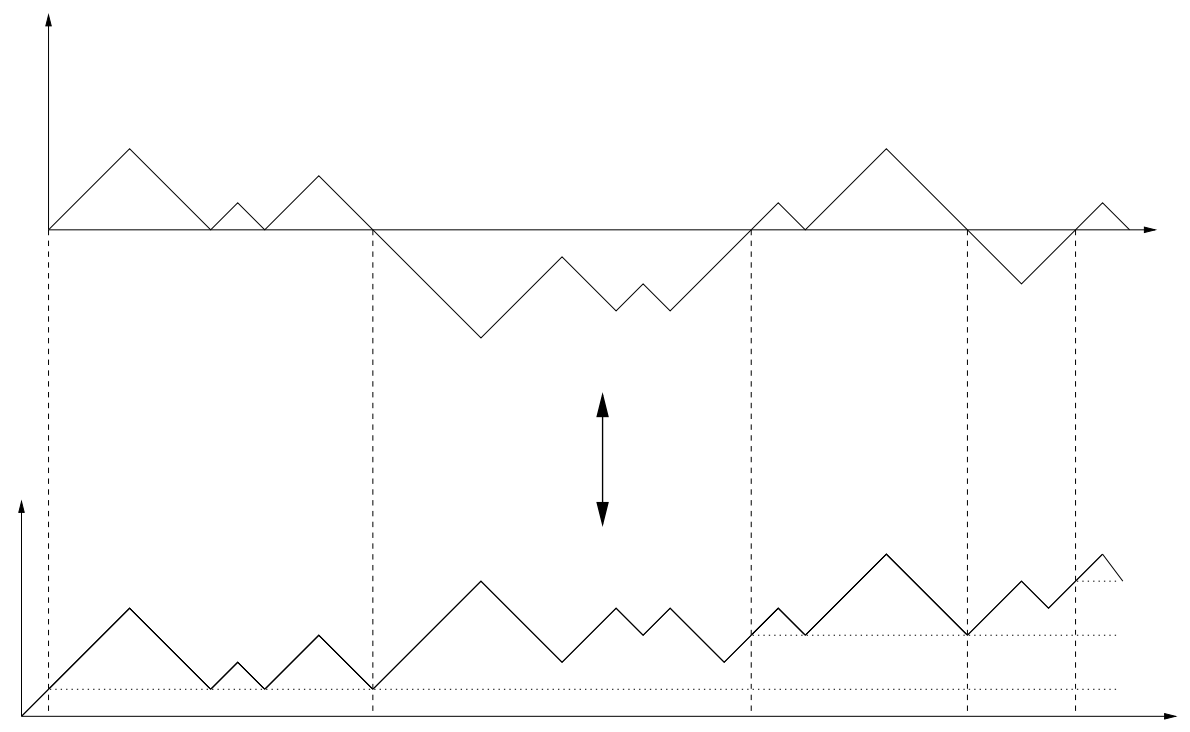

Fig. 3: The bijection between the bridge and the meander

For the other two cases of Theorem 2, recall that there is a classical bijection between the bridge and the meander. To construct a meander of length $2 n+1$ from a bridge of length $2 n$, replace each negative excursion of the bridge by its symmetric positive excursion and replace the last negative step of this symmetric positive excursion by a positive step. Finally, add a first positive step to the whole path.

Conversely, to obtain a bridge from a positive meander, remove the first step, replace every other step "visible from the right" by a negative step, so as to obtain a new positive excursion, and replace this positive excursion by the symmetric negative excursion (see Figure 3). It turns out that the algorithms in cases (1) and (3) of Theorem 2 are the image of each other by the discrete bijection.

\section{Proof of Theorems 1 and 2}

The proof that our algorithms generate random walks with the suitable distribution is quite standard, see [Mar03]. In the case of the excursion, since Rémy's algorithm generates uniform, random binary trees, and since, as noticed above, our algorithm is just an image of Rémy's algorithm by a bijection between Dyck paths and binary trees, our algorithm generates uniform, random excursions of the simple random walk. For the other cases, the idea of the proof is similar.

We establish the almost sure convergence for the excursion (case (2) in Theorem 2). The proof for the other cases is similar. The path with length $2 n$ is denoted by $\mathcal{S}^{n}$ and its normalized version by $\widehat{\mathcal{S}}^{n}$ : for $t \in[0,1]$,

$$
\widehat{\mathcal{S}}_{t}^{n}=\frac{\mathcal{S}_{[2 n t]}^{n}}{\sqrt{n}}
$$

where $[2 n t]$ stands for the integer part of $2 n t$. 


\subsection{Moving steps and local time}

Let $\left(S_{k}^{n}, S_{k+1}^{n}\right)$ be a step of $\mathcal{S}^{n}$ inserted at time $n$. We associate with this step a family $s$ of steps with exactly one step in each path $\mathcal{S}^{m}, m \geq n$. This family is defined by induction: if the step of $s$ in $\mathcal{S}^{m}$ is $\left(S_{k_{m}}^{m}, \mathcal{S}_{k_{m}+1}^{m}\right)$ then the step of $s$ in $\mathcal{S}^{m+1}$ is:

- $\left(\mathcal{S}_{k_{m}}^{m+1}, \mathcal{S}_{k_{m}+1}^{m+1}\right)$ if $\mathcal{S}^{m+1}$ is obtained from $\mathcal{S}_{m}$ by inserting two steps on the right of $k_{m}$.

- $\left(\mathcal{S}_{k_{m}+2}^{m+1}, \mathcal{S}_{k_{m}+3}^{m+1}\right)$ if $\mathcal{S}^{m+1}$ is obtained from $\mathcal{S}_{m}$ by inserting two steps on the left of $k_{m}$.

- $\left(S_{k_{m}+1}^{m+1}, S_{k_{m}+2}^{m+1}\right)$ if $\mathcal{S}^{m+1}$ is obtained from $S_{m}$ by inserting one step on the left of $k_{m}$ and one step on right of $k_{m}$.

We call such a family $s$ a moving step. We shall denote $x_{m}(s)=k_{m}$ and $y_{m}(s)=\mathcal{S}_{k_{m}}^{m}$.

If a discrete excursion $\mathcal{P}$ has length $2 n$ and $k \leq 2 n-1$, define $E_{n}(k)$ as the set of times $j \in[k, 2 n-1]$ such that

$$
\mathcal{P}(j)=\min \{\mathscr{P}(i), i \in[k, j]\}
$$

The local time for $k$ in $S^{n}$ is defined by $L_{n}(k)=\left|E_{n}(k)\right|$.

\subsection{Martingale properties}

Let $s$ be a moving step. We shall use the abridged notation $L_{n}=L_{n}\left(x_{n}(s)\right)$. Remark that if the random time $t$ chosen by the algorithm to construct $S^{n+1}$ from $S^{n}$ is in $E_{n}\left(x_{n}(s)\right)$, then $L_{n+1}-L_{n}=1$. Otherwise, if $t \notin E_{n}\left(x_{n}(s)\right), L_{n+1}=L_{n}$. As a consequence, denoting

$$
\frac{a_{n+1}}{a_{n}}=1+\frac{1}{2 n-1}
$$

and

$$
M_{n}(s)=\frac{L_{n}}{a_{n}}
$$

we check that $\left(M_{n}(s)\right)$ is a positive martingale:

$$
\begin{aligned}
& \mathbb{E} \quad\left(M_{n+1}(s) \mid M_{n}(s)\right) \\
& =\frac{L_{n}}{2 n-1}\left(\frac{L_{n}+1}{a_{n+1}}\right)+\frac{2 n-1-L_{n}}{2 n-1}\left(\frac{L_{n}}{a_{n+1}}\right) \\
& =\frac{a_{n} M_{n}(s)}{2 n-1}\left(\frac{a_{n} M_{n}(s)+1}{a_{n+1}}\right)+\frac{2 n-1-a_{n} M_{n}(s)}{2 n-1}\left(\frac{a_{n} M_{n}(s)}{a_{n+1}}\right) \\
& =\frac{a_{n} M_{n}(s)}{a_{n+1}}\left(1+\frac{1}{2 n-1}\right)=M_{n}(s)
\end{aligned}
$$

On the other hand, setting $x_{n}^{\prime}(s)=x_{n}(s) / 2 n$ an using the fact that $a_{n} \sim c \sqrt{n}$, we have

$$
\begin{aligned}
& \mathbb{E}\left(x_{n+1}^{\prime}(s) \mid x_{n}^{\prime}(s), M_{n}(s)\right) \\
& =\left(\frac{x_{n}}{2 n-1}\right)\left(\frac{x_{n}+2}{2 n+2}\right)+\left(\frac{2 n-1-x_{n}}{2 n-1}\right)\left(\frac{x_{n}}{2 n+2}\right)+O\left(\frac{L_{n}}{2 n-1} \cdot \frac{1}{n}\right) \\
& =x_{n}^{\prime}(s)+O\left(\frac{M_{n}(s)}{n^{3 / 2}}\right)
\end{aligned}
$$


which entails that $x_{n}^{\prime}(s)$ converges almost surely. We also have:

$$
\begin{aligned}
& \mathbb{E}\left(\left(M_{n+1}(s)-M_{n}(s)\right)^{2} \mid M_{n}(s)\right) \\
& =\frac{L_{n}}{2 n-1}\left(\frac{L_{n}+1}{a_{n+1}}-\frac{L_{n}}{a_{n}}\right)^{2}+\frac{2 n-1-L_{n}}{2 n-1}\left(\frac{L_{n}}{a_{n+1}}-\frac{L_{n}}{a_{n}}\right)^{2} \\
& \leq \frac{L_{n}}{2 n-1}\left(\frac{1}{a_{n+1}}\right)^{2}+\left(\frac{L_{n}}{a_{n}}\right)^{2}\left(\frac{a_{n}}{a_{n+1}}-1\right)^{2} \\
& \leq c_{1} \frac{M_{n}(s)}{n \sqrt{n}}+c_{2} \frac{M_{n}(s)^{2}}{n^{2}}
\end{aligned}
$$

where $c_{1}, c_{2}$ are universal constants which do not depend on $s$. Using a maximal inequality we obtain

$$
\mathbb{E}\left(\sup _{n \geq N}\left(M_{N}(s)-M_{n}(s)\right)^{2}\right) \leq \frac{c_{3} M_{N}(s)}{\sqrt{N}}
$$

where again $c_{3}$ does not depend on $s$. Similarly,

$$
\begin{aligned}
& \mathbb{E}\left(\left(x_{n+1}^{\prime}(s)-x_{n}^{\prime}(s)\right)^{2} \mid x_{n}^{\prime}(s), M_{n}(s)\right) \\
& \leq\left(\frac{x_{n}}{2 n-1}\right)\left(\frac{x_{n}+2}{2 n+2}-\frac{x_{n}}{2 n}\right)^{2}+\left(\frac{2 n-1-x_{n}}{2 n-1}\right)\left(\frac{x_{n}}{2 n+2}-\frac{x_{n}}{2 n}\right)^{2}+O\left(\frac{L_{n}}{2 n-1} \cdot \frac{1}{n^{2}}\right) \\
& \leq \frac{c_{4}}{n^{2}}
\end{aligned}
$$

and

$$
\mathbb{E}\left(\sup _{n \geq N}\left(x_{N}^{\prime}(s)-x_{n}^{\prime}(s)\right)^{2}\right) \leq \frac{c_{5}}{N}
$$

\subsection{Strong convergence}

The key argument for the proof of strong convergence is the following: For every moving step $s$,

$$
\left(\frac{x_{n}(s)}{2 n}, \frac{S_{x_{n}(s)}^{n}}{\sqrt{n}}\right)
$$

converges almost surely as $n \rightarrow \infty$ to some point $(X(s), Y(s)) \in \mathbb{R}^{2}$.

The convergence of $x_{n}^{\prime}(s)=x_{n}(s) / 2 n$ was established in Section 3.2. In fact, $x_{n}(s) / 2$ is almost a Pólya urn, up to a small perturbation due to $L_{n}$. On the other hand, as the martingale $M_{n}(s)$ converges almost surely and as $a_{n} \sim c \sqrt{n}$, the quantity $L_{n} / \sqrt{n}$ converges almost surely to some real $Y^{\prime}(s)$. Moreover, each time $L_{n}$ increases, $S_{x_{n}(s)}^{n}$ either increases with probability $1 / 2$ or remains constant with probability $1 / 2$, independently of the past. Hence $S_{x_{n}(s)}^{n}$ is the sum of $L^{n}$ independent Bernoulli random variables and by the law of large numbers, almost surely,

$$
\frac{S_{x_{n}(s)}^{n}}{\sqrt{n}} \rightarrow \frac{Y^{\prime}(s)}{2}
$$

This way we get a discrete "skeleton" $\cup_{s}\{(X(s), Y(s)\}$, where the union is over all moving steps. If we denote by $\mathbf{B}$ the closure in $\mathbb{R}^{2}$ of $\cup_{s}\left\{(X(s), Y(s)\}\right.$, then the curves $\widehat{\mathcal{S}}^{n}$ converge (in some sense) to B. It 
should be clear that $\mathbf{B}$ is the graph of a Brownian excursion $\left(B_{t}, 0 \leq t \leq 1\right)$ and that for every $t, \widehat{\mathcal{S}}_{t}^{n} \rightarrow B_{t}$. Moreover, one can prove that $\sup _{t}\left|B_{t}-\widehat{\mathcal{S}}_{t}^{n}\right| \rightarrow 0$ almost surely. Technical details can be found in [Mar03]. Let us bound

$$
I_{n}=\mathbb{E}\left[\int_{0}^{1} d\left(\left(t, \widehat{S}_{t}^{n}\right), \mathbf{B}\right) d t\right]
$$

where $d(.,$.$) stands for the euclidean distance in \mathbb{R}^{2}$. We have

$$
I_{n}=\sum_{k=1}^{n} \mathbb{E}\left[\int_{(k-1) / n}^{k / n} d\left(\left(t, \widehat{\mathcal{S}}_{t}^{n}\right), \mathbf{B}\right) d t\right]
$$

and for every $1 \leq k \leq n$,

$$
\int_{(k-1) / n}^{k / n} d\left(\left(t, \widehat{\mathcal{S}}_{t}^{n}\right), \mathbf{B}\right) d t \leq \int_{(k-1) / n}^{k / n} d\left(\left(t, \widehat{\mathcal{S}}_{t}^{n}\right),\left(X\left(s_{t}\right), Y\left(s_{t}\right)\right)\right) d t
$$

where $s_{t}$ is the moving step containing the step $\left(\mathcal{S}_{k-1}^{n}, S_{k}^{n}\right)$ in $S^{n}$. As a consequence of 2 and 3 one easily obtains

$$
\mathbb{E}\left[\int_{(k-1) / n}^{k / n} d\left(\left(t, \widehat{\mathcal{S}}_{t}^{n}\right), \mathbf{B}\right) d t \mid \widehat{\mathcal{S}}^{n}\right] \leq \frac{c_{6}}{n} \cdot \frac{\widehat{\mathcal{S}}_{k / n}^{n}}{n^{1 / 4}}
$$

Summing over $k$ and integrating with respect to the law of $\widehat{\mathcal{S}}^{n}$,

$$
I_{n} \leq c_{6} \mathbb{E}\left[\sup _{t} \frac{\widehat{\mathcal{S}}_{t}^{n}}{n^{1 / 4}}\right] \leq \frac{c_{7}}{n^{1 / 4}}
$$

since by Donsker's theorem, $\mathbb{E}\left[\sup _{t}\left(\widehat{\mathcal{S}}_{t}^{n}\right)\right]$ is bounded in $n$. Formula 1 follows from 4 and from the fact that a Brownian excursion is almost surely Hölder-continuous with index $1 / 2-\varepsilon$ for every $\varepsilon>0$.

\section{Further results}

\subsection{Random partitions}

Recall that a partition is exchangeable if its law is invariant by permutation. The Chinese restaurant is a model parametrized by two reals $0 \leq \alpha<1$ and $\theta>-\alpha$, generating an exchangeable partition. Imagine a restaurant with infinitely many tables and infinitely many customers, the first customer sitting at the first table. The seating plan is defined by induction as follows.

Suppose that at a given moment, $n$ customers have arrived and occupy $k$ tables, the number of customers at each table being $n_{1} \ldots n_{k}$ respectively with $n_{1}+\ldots+n_{k}=n$. Then the $(n+1)$-th customer sits at table number $i, 1 \leq i \leq k$, with probability $\left(n_{i}-\alpha\right) /(n+\theta)$, and at table number $k+1$ with probability $(k \alpha+\theta) /(n+\theta)$. See Pitman [Pitar] for a detailed account.

Associate with this process a partition of $\mathbb{N}$ by saying that $i$ and $j$ are in the same block of the partition if and only if the $i$-th and $j$-th customers sit at the same table. Then one can check that this random partition is exchangeable.

The set of return times to 0 of a simple random walk also generates a random partition $\mathcal{P}$. Indeed, consider a random walk of length $2 n$ and say that two integers $1 \leq i, j \leq n$ are in the same block if there 
is no zero of the random walk between times $2 i-1$ and $2 j-1$. Then construct the exchangeable partition $\mathcal{P}^{\prime}$ by choosing a random, uniform permutation $\sigma$ on $\{1,2 \ldots n\}$ and saying that $i$ and $j$ are in the same block of $\mathcal{P}^{\prime}$ if and only if $\sigma(i)$ and $\sigma(j)$ are in the same block of $\mathcal{P}$. A link with the Chinese restaurant is the following result [PY97]:

Theorem 4 (Pitman-Yor) (i) The exchangeable partition obtained from a simple random walk of length $2 n$ has the same law as the exchangeable partition obtained from the first $n$ customers of a Chinese restaurant with parameters $(1 / 2,0)$.

(ii) The exchangeable partition obtained from a simple random walk of length $2 n$ conditioned to return to 0 at time $2 n$ has the same law as the exchangeable partition obtained from the first $n$ customers of a Chinese restaurant with parameters $(1 / 2,1 / 2)$.

Alternatively, this theorem can be viewed as a direct corollary of Theorem 1 and Case (1) of Theorem 2. Indeed, at each iteration of the algorithm, two steps are added and either they are incorporated to an excursion or to the meander, or these two steps form a new excursion, and the respective probabilities correspond to those given by the Chinese restaurant. Remark that this way, we have embedded the Chinese restaurant in our construction.

\subsection{Proof of Theorem 3}

Let us turn our attention to Theorem 3. First remark that by the same argument as in the previous subsection, one can embed the Chinese restaurant with parameters $(1 / 2,0)$ in the construction given by the algorithm of Theorem 3 . We represent this restaurant by a function $f: \mathbb{N} \rightarrow \mathbb{N}$, where $f(n)=k$ means that the $n$-th customer sits at the $k$-th table.

Moreover, recall [Pitar] that if a partition is exchangeable, each block $B$ almost surely has an asymptotic frequency, which is the limit of $|B \cap\{1,2, \ldots n\}| / n$, and that the law of these asymptotic frequencies determines the law of the exchangeable partition.

Define the uniform total order on a countable set $S$ as a random total order $\prec$ where, for each finite subset $S^{\prime}$ of $S$, the restriction of $\prec$ to $S^{\prime}$ is uniformly distributed on $S^{\prime}$ among all possible total orders. For instance, the natural order on $\mathbb{R}$ induces a total order on the set of excursions of the random walk: if $e, e^{\prime}$ are two excursions, say that $e \prec e^{\prime}$ if $e$ occurs before $e^{\prime}$. Then one easily checks that $\prec$ is a uniform total order, independent of $f$.

Using the same arguments as in the proof of (2) in Theorem 2, each excursion $e$ of the random walk constructed by the algorithm converges almost surely to a Brownian excursion. Hence the sequence of random walks of Theorem 3 converges almost surely to a continuous function $\beta$ with the following properties:

- $\beta$ is a concatenation of Brownian excursions,

- The family of lengths of these excursions has the same law as the family of asymptotic frequencies of a Chinese restaurant with parameters $(1 / 2,0)$.

- The order $\prec$ on these excursions is a uniform total order.

It follows (see for instance [Pitar]) that $\beta$ is a normalized pseudobridge. 


\section{Acknowledgements}

My interest on this subject was raised by Jim Pitmans's course in St-Flour. I thank Jean-François Marckert and Philippe Duchon for references.

\section{References}

[Don51] Monroe D. Donsker. An invariance principle for certain probability limit theorems. Mem. Amer. Math. Soc., 1951(6):12, 1951.

[KMT75] J. Komlós, P. Major, and G. Tusnády. An approximation of partial sums of independent RV's and the sample DF. I. Z. Wahrscheinlichkeitstheorie und Verw. Gebiete, 32:111-131, 1975.

[KMT76] J. Komlós, P. Major, and G. Tusnády. An approximation of partial sums of independent RV's, and the sample DF. II. Z. Wahrscheinlichkeitstheorie und Verw. Gebiete, 34(1):33-58, 1976.

[Mar03] P. Marchal. Almost sure convergence of the simple random walk to brownian motion. In preparation, 2003.

[Pitar] Jim Pitman. Combinatorial stochastic processes. Springer-Verlag, To appear.

[PY97] Jim Pitman and Marc Yor. The two-parameter Poisson-Dirichlet distribution derived from a stable subordinator. Ann. Probab., 25(2):855-900, 1997.

[Rém85] Jean-Luc Rémy. Un procédé itératif de dénombrement d'arbres binaires et son application à leur génération aléatoire. RAIRO Inform. Théor., 19(2):179-195, 1985. 